Artículo

\title{
Propiedades fisicoquímicas de frutos silvestres de Rubus con potencial nutracéutico y alimenticio
}

\author{
Rubio Ochoa Eréndira ${ }^{1}$ \\ Pérez Sánchez Rosa Elena ${ }^{3}$ \\ Ávila Val Teresa C. ${ }^{2}$ \\ Gómez Leyva Juan Florencio ${ }^{4}$ \\ García Saucedo Pedro Antonio ${ }^{1,2}$
}

${ }^{1}$ Facultad de Agrobiología ‘Presidente Juárez'-Universidad Michoacana de San Nicolás de Hidalgo. Paseo Lázaro Cárdenas 2290, Emiliano Zapata, Melchor Ocampo, Uruapan, Michoacán. CP. 60170. (ere.rub.och@hotmail.com). ${ }^{2}$ Facultad de Agrobiología-Universidad Michoacana de San Nicolás de Hidalgo. Paseo Lázaro Cárdenas 2290, Emiliano Zapata, Melchor Ocampo, Uruapan, Michoacán. CP. 60170. (tereavilaval@yahoo.com.mx). ${ }^{3}$ Facultad de Químico Farmacobiológicas. Avenida Tzintzuntzan 173, Matamoros, Morelia, Michoacán. CP. 58240. (rosa_elenap@yahoo.com). ${ }^{4}$ Instituto Tecnológico de Tlajomulco-TecNM. Carretera a San Miguel de Cuyutlán km 10, Tlajomulco de Zúñiga, Jalisco. CP. 45640. (jfgleyva@hotmail.com).

Autor para correspondencia: garsapan@ hotmail.com.

\section{Resumen}

Las especies del género Rubus, comúnmente son explotados para su consumo en fresco, gastronomía regional y herbolaria tradicional, dichos beneficios son atribuidos a la presencia y acción de sus metabolitos polifenólicos como los flavonoides y antocianinas, que son conocidos por su actividad antidiabética, anticancerígena, etc. En México se reportan alrededor de 15 especies silvestres del género Rubus. No obstante, el crecimiento poblacional ha invadido su territorio, comprometiendo su desarrollo. Por lo anterior y con el fin de rescatar e incorporar en programas de mejoramiento genético los materiales silvestres, el objetivo del presente trabajo fue evaluar la composición fisicoquímica y actividad antioxidante en frutos de tres especies silvestres (Rubus adenotrichus, Rubus pringlei y Rubus glaucus Beth), comparados contra una variedad comercial (Tupy). Se colectaron frutos en el estado de Michoacán y se evaluaron parámetros físicoquímicos, polifenoles, flavonoides, antocianinas y capacidad antioxidante (CA) en extractos etanólicos. En los resultados, la longitud del fruto vario de $1.7-2.23 \mathrm{~cm}$ y el peso unitario de 1.1-3.1 g, la relación de sólidos solubles totales y acidez titulable de 8.32-14.76. Los polifenoles totales reportaron datos de 285.06-592.61 mg EAG/100 g PF, flavonoides totales de 93.13-36.4 mg EQ/100 g PF y antocianinas de 18.43-4.32 $\mathrm{mg} \mathrm{L}^{-1}$. La CPA de 65.7-25.15 $\mu \mathrm{M}$ ET/g PF. Este estudio demostró que las especies silvestres cumplen con características fisicoquímicas y nutracéuticas para ser incorporadas en programas de fitomejoramiento o la industria farmacéutica.

Palabras claves: capacidad antioxidante, flavonoides, polifenoles.

Recibido: abril de 2019

Aceptado: julio de 2019 


\section{Introducción}

El género Rubus cuenta con 12 subgéneros y un estimado de 750 a 1000 especies (Tatjana et al., 2010; Moreno et al., 2018). Como miembros representativos de este género, se encuentran las frambuesas y zarzamoras, conocidas por sus frutos comestibles, los cuáles son clasificados como polidrupas, ya que son agregados de pequeños frutos carnosos denominados como drupelas y cada una con semilla, reunidas alrededor de un eje común. En estadios inmaduros las drupelas suelen ser de color verde y a medida que maduran se tornan de color rojizo, en caso de las frambuesas o hasta llegar a un morado intenso y brillante en las zarzamoras (Hummer et al., 2017).

Principalmente se desarrollan en zonas frías y templadas de América, Europa, norte de África y noroeste de Asia, considerando a China el lugar donde se concentra la mayor diversidad de especies de Rubus en el mundo (Graham et al., 2011). En general, las zarzamoras son consideradas como especies con amplia capacidad de adaptación a condiciones ambientales, por lo que, algunas son catalogadas como hierba invasiva, promoviendo su eliminación y amenazando la pérdida de especies silvestres.

Aunado a esto, las implicaciones actuales del cambio climático influyen sobre los ejemplares silvestres, lo que representan la pérdida de un recurso genético con alto potencial para ser utilizado dentro de programas para el desarrollo de cultivares mejor adaptados a los cambios de su entorno (Graham et al., 2011), siendo las especies silvestres mexicanas un recurso poco investigado para la explotación comercial.

En los últimos treinta años la producción de zarzamora y frambuesa han tenido un crecimiento considerable, ya que en 1990 la extensión cultivada en Norteamérica fue de 4385 ha, aportando el 75\% el noroeste del Pacífico, una década después, Centroamérica se convirtió en la zona productora más grande del mundo y su producción mayoritariamente se destinaba para comercializar en fresco y exportar a mercados minoristas en Estados Unidos y Europa, (Clark et al., 2011), once años más tarde, se calcularon alrededor de 20035 ha de zarzamoras cultivadas comercialmente en todo el mundo, además de 8000 ha de fruta cosechada en condiciones silvestre para una producción total de $140292 \mathrm{t}$.

Para 2014 se estimaron más de 25000 ha cultivadas en el mundo, considerando a México con la mayor extensión; así como, con la mayor producción de zarzamoras con un total de 248517 t, seguido de Colombia con 110453 t e Italia con 107479 t. Debido a lo anterior, ha surgido la búsqueda continua de especies mejoradas con características atractivas para el productor y consumidor, por ejemplo la variedad 'Tupy' y 'Brazos'; no obstante, en países como Colombia y al igual que casi todas las regiones del mundo donde Rubus es nativo, han desarrollado prósperas industrias basadas en sus especies locales (Ayala et al., 2013), no así para el caso de México.

En México se reportan alrededor de 15 especies silvestres distribuidas en el territorio nacional (Segura et al., 2009). Dentro del estado de Michoacán, se han encontrado diferentes especies de zarzamoras y frambuesas silvestres, principalmente en los municipios de la meseta purépecha, donde sus frutos son colectados para comercializarlos en mercados regionales para su consumo en fresco, aunque comúnmente son incorporados en la gastronomía regional, ya sea en elaboración de tamales o bebidas refrescantes y fermentadas. 
Por otro lado, sus hojas y tallos se han utilizado dentro de la herbolaria tradicional para curar algunas afecciones como gripe, náuseas durante el embarazo, malestares menstruales y facilitar el parto, además la fruta se considera un laxante suave si se come en grandes cantidades (Hummer et $a l ., 2010)$. Estos beneficios en la salud son atribuidos a la presencia y acción de sus metabolitos polifenólicos como los flavonoides y antocianinas (Cuevas et al., 2010), que comúnmente son conocidos por su actividad antidiabética, anticancerígena, antimicrobiana, antiinflamatoria y su destacada capacidad antioxidante (Azofeifa et al., 2013).

Por lo anterior y con el fin de rescatar, estudiar e incorporar en programas de mejoramiento genético los materiales silvestres mexicanos, en el presente trabajo se tuvo como objetivo evaluar la composición fisicoquímica y actividad antioxidante en frutos de tres especies silvestres del género Rubus, comparados contra la variedad comercial Tupy.

\section{Materiales y métodos}

\section{Material biológico}

Los frutos de zarzamoras y frambuesa silvestre fueron recolectados en los municipios de Uruapan y Nahuatzen en el estado de Michoacán, México. Los sitios de colecta fueron referenciados mediante coordenadas geográficas para cada especie, mismas que se identificaron mediante biología molecular en el Laboratorio de Biología Molecular del Instituto Tecnológico de Tlajomulco, Jalisco, México.

Las especies fueron reconocidas como Rubus adenotrichus (1 $815 \mathrm{msnm}$; 19 $26^{\circ}$ '48' latitud norte, $102^{\circ}$ 4' 38' ' longitud oeste), Rubus pringlei (2 $769 \mathrm{msnm}, 19^{\circ} 40^{\prime} 30^{\prime}$ ' latitud norte; $101^{\circ}$ 50' 8', Longitud Oeste), Rubus glaucus Beth (2 769 msnm; 19 40' 29' ' latitud norte; $101^{\circ} 50^{\prime}$ 9' ' longitud oeste) y la variedad comercial Tupy (1 210 msnm; 19 39' 16' latitud norte; 101 91' 50', longitud oeste). Los frutos se seleccionaron en madures de consumo según su coloración. Al momento de la colecta se registró el tamaño del fruto mediante un Vernier (Truper análogos estándar), número de drupillas y el peso en gramos con una balanza digital (Sartorius, modelo BL210S).

\section{Composición proximal fisicoquímica}

Los parámetros fisicoquímicos fueron evaluados en fruta fresca, mediante la aplicación de normas oficiales de análisis para: porcentaje de humedad (AOAC 934,06/2007), acidez titulable expresada en ácido cítrico (AOAC 942,15/2007), pH por potenciometría (AOAC 981, 12/2002), sólidos solubles totales ( ${ }^{\circ}$ Brix) con un refractómetro de escala 0-30 (Atago, modelo master-BX/S28M); asimismo, se evaluaron los azucares reductores según el método de ácido 3,5-dinitrosalicílico (DNS) (Amid et al., 2014), que consistió en la preparación del reactivo DNS disolviendo $75 \mathrm{~g}$ de tartrato de sodio y potasio tetrahidratado en agua destilada, agregando $50 \mathrm{~mL}$ de hidróxido de sodio $2 \mathrm{M}$ y $75 \mathrm{~mL}$ de agua destilada caliente, finalmente se añadieron $0.25 \mathrm{~g}$ de ácido 3,5dinitrosalicílico, la reacción se llevó a cabo con $100 \mu \mathrm{L}$ del extracto, adicionando $1 \mathrm{~mL}$ del reactivo DNS e incubando durante $10 \mathrm{~min}$. La absorbancia se determinó a $570 \mathrm{~nm}$ y los resultados se expresaron en gramos de fructosa por $100 \mathrm{~g}$ de muestra. 


\section{Cuantificación de polifenoles, flavonoides, antocianinas totales y capacidad antioxidante}

La extracción se realizó por maceración a partir de $1 \mathrm{~g}$ de fruta fresca con $9 \mathrm{~mL}$ de etanol al 80\%, por $48 \mathrm{~h} \mathrm{a} 4{ }^{\circ} \mathrm{C}$ en ausencia de luz. La mezcla se centrifugó a $13000 \mathrm{rpm}$ por $10 \mathrm{~min}$, se recuperó el sobrenadante y se utilizó para la cuantificación de polifenoles totales (PT), flavonoides totales (FT), antocianinas totales (ANT) y capacidad antioxidante (CA).

Los PT se cuantificaron mediante lo reportado por Zielinsli y Kozolwaska, (2000). Se tomó $50 \mu \mathrm{L}$ de muestra, se mezcló con $200 \mu \mathrm{L}$ de agua destilada y $250 \mu \mathrm{L}$ de reactivo de Folin-Ciocalteu al 1 $\mathrm{N}$, transcurridos tres minutos, se agregaron $500 \mu \mathrm{L}$ de $\mathrm{Na}_{2} \mathrm{CO}_{3}$ al 7.5\%. Se incubó durante 15 min y se registró la absorbancia a $760 \mathrm{~nm}$. Los resultados se expresaron en equivalentes de ácido gálico por cien gramos de peso fresco $(\mathrm{mg}$ EAG/100 g PF). Se realizó una curva de calibración con ácido gálico a diferentes concentraciones $0.01-0.50 \mathrm{mg} \mathrm{mL}^{-1}$ (diez puntos de datos $\mathrm{R}^{2}=0.998$ ).

Los FT se evaluaron mediante lo reportado por Chang et al. (2002), mezclando $100 \mu \mathrm{L}$ del extracto con $200 \mu \mathrm{L}$ de acetato de potasio $1 \mathrm{M}, 200 \mu \mathrm{L}$ de nitrato de aluminio al $10 \%$ y $1 \mathrm{~mL}$ de etanol al $80 \%$, posteriormente se incubó durante 40 min y se leyó la absorbancia a $415 \mathrm{~nm}$. Los resultados se reportaron en equivalentes de quercetina por cien gramos de peso fresco (mg EQ/100 g PF). La curva de calibración fue calculada a diferentes concentraciones de quercetina que incluyeron diez puntos entre 0.001-0.01 $\mathrm{mg} \mathrm{mL}^{-1}\left(\mathrm{R}^{2}=0.998\right)$.

Para las ANT se basó en el método diferencial de pH, según Wrolstad et al. (2005). Se prepararon dos soluciones amortiguadoras, una con $\mathrm{pH} 1$ de cloruro de potasio a $0.025 \mathrm{M}$ y la segunda con $\mathrm{pH}$ 4.5 de acetato de sodio a $0.4 \mathrm{M}$. En ambas soluciones se ajustó el pH con $\mathrm{HCl}$ concentrado. Se tomaron $20 \mu \mathrm{L}$ del extracto y se completó a $1.5 \mathrm{~mL}$ con la solución respectiva. La absorbancia de cada solución se registró a 520 y 700 nm. La concentración de antocianinas totales se determinó mediante la siguiente ecuación:

Antocianinas totales $\mathrm{mg} \mathrm{g}^{-1}=\frac{\mathrm{A} \times \mathrm{MW} \times \mathrm{DF} \times 1000}{\sum \times 1}$

Donde: $\mathrm{MW}=$ peso molecular de cyanidin-3-glucósido (449.2 $\left.\mathrm{g} \mathrm{mol}^{-1}\right) ; \mathrm{DF}=$ factor de dilución (75) y $\Sigma=$ coeficiente de extinción molar (26 900) y el valor de A se obtuvo con la ecuación: A= (Abs 520 -Abs 700) pH 1 (Abs 520-Abs 700) pH 4.5. Los resultados se expresaron en $\mathrm{mg}$ de antocianinas totales por cien gramos de peso fresco $(\mathrm{mg} / 100 \mathrm{~g} \mathrm{PF})$.

La CA se realizó por el método del radical DPPH (1,1-diphenyl-2-picrylhydrazyl), acorde a lo reportado por Brand et al. (1995). Para ello, se disolvió $150 \mathrm{mM}$ DPPH en metanol al 80\%. Se tomó una alícuota de $100 \mu \mathrm{L}$ del extracto de cada muestra y se añadieron $900 \mu \mathrm{L}$ de solución DPPH. Las mezclas fueron incubadas por $30 \mathrm{~min}$ y se determinó su absorbancia a $515 \mathrm{~nm}$. Los resultados se expresaron como micromolar equivalente de trolox por gramo de peso fresco ( $\mu \mathrm{mol} \mathrm{ET/g} \mathrm{PF).}$ La curva de calibración fue calculada a diferentes concentraciones de trolox que incluyeron diez puntos entre $1 \mathrm{a} 1.500 \mu \mathrm{M} \mathrm{mL} \mathrm{m}^{-1}\left(\mathrm{R}^{2}=0.998\right)$.

\section{Análisis estadístico}

Los análisis estadísticos se realizaron con el paquete estadístico Statgraphics Centurion Versión XV.II. Se reportaron los valores medios y sus desviaciones estándar. Se realizaron análisis de varianza de una vía, se comparó las medias múltiples mediante la prueba de Tukey $(p \leq 0.05)$. 


\section{Resultados}

En el Cuadro 1, se muestran las principales características morfológicas distintivas de cada material biológico utilizado en el presente estudio, las cuales se describen a continuación.

Cuadro 1. Características morfologías de la flor, tallo y fruto de especies silvestres de Rubus.

\begin{tabular}{|c|c|c|c|}
\hline Especie & Flor & tallo & Fruto maduro \\
\hline R. adenotrichus & & & \\
\hline R. glaucus Beth & & & \\
\hline R. pringlei & & & \\
\hline Variedad Tupy & & & \\
\hline
\end{tabular}


R. adenotrichus (zarzamora): tiene un tallo suberecto, circular angulosa, cubierto por un gran número de pelos glandulares de coloración rojiza; muestra un hábito de crecimiento trepador con aguijones separados, sus hojas son dentadas con haz verde obscuro, además su inflorescencia es piramidal con flores de color rosa pálido, como resultado racimos que producen de 70 a 150 frutos.

R. glaucus Beth (zarzamora): presenta un tallo erecto y circular con numerosas espinas y cubierto por una fina capa de cera blanquecina conocida como pruina, sus hojas presentan foliolos de forma laminar y suorbicular, su inflorescencia se da en forma de roseta cuyas flores presentan pétalos de coloración blanquecina, pequeños y separados; sus racimos muestran entre 6 y 8 frutos.

R. pringlei (frambuesa): su tallo principal tiene un hábito de crecimiento erecto, circular y de color rojizo. Sus hojas son pinnaticompuestas con 3-7 foliolos, obovadas, aserrada con base cordada, muestran un color verde por el haz y blanquecinas aterciopeladas por el envés. Las flores tienen una corola compuesta de 5 pétalos blancos y poseen numerosos estambres y pistilos dando lugar a frutos de color rojo en estadio de madurez; sus racimos generan entre 2 y 5 frutos.

Variedad Tupy: es un híbrido generado a partir de la cruza entre la variedad 'Comanche' y una selección de Uruguay. Esta variedad presentó tallo con numerosas espinas cortas y afiladas, cuyo hábito de crecimiento es erecto, sus hojas son grandes y palmeadas, cuenta con flores de cinco pétalos blanquecinas de 2 a $3 \mathrm{~cm}$ de diámetro.

\section{Composición proximal fisicoquímica}

Los resultados de los análisis fisicoquímicos efectuados a las especies silvestre del género Rubus, evidencian variaciones en cuanto a la longitud del fruto que va de 1.7 a $2.23 \mathrm{~cm}$ (Cuadro 2). Por otro lado, se registró de 34 a 89 en el número de drupillas, siendo la especie $R$. pringlei la de menor cantidad y Rubus adenotrichus quien reportó el mayor número de durpillas. Referente a la relación entre longitud y número de drupillas, se observó que entre mayor longitud del fruto menor cantidad de drupillas. El peso unitario varió entre 1.1-3.1 g siendo $R$. pringlei la de menor peso y $R$. glaucus Beth con el valor estadísticamente más alto.

Cuadro 2. Composición proximal de frutos maduros de zarzamoras ( \pm desviación estándar).

\begin{tabular}{lcccc}
\hline Parámetro & R. adenotrichus & R. glaucus Beth & R. pringlei & Variedad Tupy \\
\hline Longitud (cm) & $2.23 \pm 0.15 \mathrm{bc}$ & $2.7 \pm 0.26 \mathrm{~b}$ & $1.7 \pm 0.15 \mathrm{c}$ & $4.6 \pm 0.36 \mathrm{a}$ \\
Núm. de drupillas & $89 \pm 3.6 \mathrm{~b}$ & $70.3 \pm 2.08 \mathrm{~b}$ & $34.6 \pm 4.5 \mathrm{~d}$ & $64 \pm 4.58 \mathrm{c}$ \\
Peso (g) & $2.5 \pm 0.27 \mathrm{c}$ & $3.1 \pm 0.1 \mathrm{~b}$ & $1.1 \pm 0.1 \mathrm{~d}$ & $7.2 \pm 0.2 \mathrm{a}$ \\
$\mathrm{pH}$ & $3.1 \pm 0.08 \mathrm{~b}$ & $3.2 \pm 0.02 \mathrm{~b}$ & $3.3 \pm 0.02 \mathrm{a}$ & $3.3 \pm 0.01 \mathrm{a}$ \\
Humedad (\%) & $75.1 \pm 0.04 \mathrm{~d}$ & $86.89 \pm 0.1 \mathrm{~b}$ & $82.6 \pm 0.02 \mathrm{c}$ & $87.7 \pm 0.03 \mathrm{a}$ \\
Azúcares reductores & $1.34 \pm 0.02 \mathrm{~b}$ & $1.55 \pm 0.1 \mathrm{a}$ & $1.48 \pm 0.2 \mathrm{a}$ & $1.56 \pm 0.15 \mathrm{a}$ \\
Acidez titulable (\% de ácido cítrico) & $0.8 \pm 0.02 \mathrm{c}$ & $0.90 \pm 0.03 \mathrm{bc}$ & $1.05 \pm 0.12 \mathrm{ab}$ & $1.1 \pm 0.09 \mathrm{a}$ \\
Sólidos solubles totales ( ${ }^{\circ}$ Brix) & $12.4 \pm 0.19 \mathrm{a}$ & $7.6 \pm 0.10 \mathrm{~d}$ & $10.7 \pm 0.1 \mathrm{~b}$ & $9.8 \pm 0.09 \mathrm{c}$ \\
$\begin{array}{l}\text { Relación sólidos } \text { solubles/acidez } \\
\text { titulable }\end{array}$ & $14.76 \pm 0.41 \mathrm{a}$ & $8.32 \pm 0.14 \mathrm{c}$ & $10.24 \pm 1.08 \mathrm{bc}$ & $8.96 \pm 0.9 \mathrm{c}$ \\
\hline
\end{tabular}

Promedio con letras distintas indican diferencias significativas entre filas $(p \leq 0.05)$. 
En cuanto al contenido de humedad, destaco R. glaucus Beth, mostrando diferencia significativa con respecto a las otras especies silvestres. Si bien se sabe que la elevada humedad en frutas y hortalizas es una desventaja ya que, al existir mayor disponibilidad de agua libre se disminuye la vida de anaquel, de igual manera se reduce la concentración de azúcares y por tanto la dulzura del producto. Por lo que las silvestres al poseer porcentajes menores de humedad se prevé una mayor vida de anaquel que la variedad comercial Tupy.

El contenido de sólidos solubles totales en las cuatro especies presentó diferencias estadísticas significativas; sin embargo, se observaron los valores más alto para las especies silvestres $R$. adenotrichus y $R$. pringlei con $12.4 \pm 0.19$ y $10.7 \pm 0.10^{\circ}$ Brix, en contraste la variedad Tupy reportó $9.8 \pm 0.09{ }^{\circ}$ Brix, mientras Rubus glaucus Beth obtuvo la menor cantidad de sólidos solubles (7.6 $\pm 0.1^{\circ}$ Brix) en comparación con la variedad comercial.

El alto contenido de sólidos solubles totales es reflejado en el dulzor de la fruta, característica calificada e incorporada en la elaboración de vinos y licores (Coronel, 2008). En cuanto al porcentaje de acidez titulable se encontraron valores de 0.8-1.1 y las especies silvestres mostraron porcentajes de acidez estadísticamente menores, respecto a la variedad comercial.

En los frutos a medida que avanza la maduración los ácidos orgánicos son respirados o convertidos en azúcares, disminuyendo su contenido (Seymour et al., 1993), por lo que su sabor no solo se ve afectado por el contenido de azúcares, sino también por la presencia de compuestos volátiles y ácidos orgánicos, siendo el ácido cítrico, málico y tartárico los más abundantes en frutos de zarzamoras y frambuesas, constituyendo el ácido cítrico entre 30-95\% respecto al total de ácidos orgánicos (Petkovsek, et al., 2012).

Algunos ácidos orgánicos como es el ácido cítrico, han sido ampliamente incorporados en la industria alimentaria, debido a que se desempeña como regulador de acidez, provee firmeza, realza el sabor, actúa como conservador y antioxidantes, además de que brinda estabilidad a lípidos y antioxidantes primarios como ácidos fenólicos (ácido gálico y ferúlico) y son capaces de inactivar a iones metálicos que actúan como prooxidantes, tal es el caso del hierro y cobre mediante la formación de quelatos (Quitmann et al., 2014).

La relación de sólidos solubles totales y acidez titulable de $R$. adenotrichus reportó $14.76 \pm 0.41$, se incluyó como el valor más alto en frutos silvestres de Rubus hirsutus Thunb con relaciones que van de 31.31-11.23. Si bien la relación obtenida en las especies de Rubus glaucus y pringlei fueron estadísticamente menores, respecto a $R$. adenotrichus, se encuentran dentro de lo reportado Lewers et al. (2010) en 21 variedades comerciales de zarzamoras y frambuesas (4.8-11.9). Lewers et al. (2010) hacen referencia de un sabor más agradable para fresa con una relación alrededor 10, a su vez esto podría lograrse con niveles altos a moderados de sólidos solubles totales y baja acidez titulable, como en el caso de las especies silvestres del presente trabajo.

Una relación superior a 12.5, es considerada una característica apreciada en un sabor dulce para el consumidor. Por otro lado, Vergara et al. (2016) hacen referencia que la industria de los Estados Unidos de América prefiere zarzamoras de buen color con alto contenido de sólidos solubles y acidez, al igual se buscan con bajo número de semillas y $\mathrm{pH}$, mientras que, para los consumidores, las cualidades, como dulzura, acidez, astringencia, color, firmeza y ausencia de semillas son importantes tanto para procesados y frutas frescas. 
Estas características permiten considerar a las especies silvestres como candidatas para su incorporación a programas de manejo agronómico que permita incrementar el tamaño del fruto, así como a procesos de cruce entre especies, ya que ha beneficiado la obtención de frutos grandes con un número moderado de semillas (Clark et al., 2011).

\section{Contenido de polifenoles, flavonoides, antocianinas y capacidad antioxidante}

Los valores registrados de polifenoles totales para los materiales estudiados reportaron datos que van de $285.06 \pm 8.49$ a $592.61 \pm 7.03 \mathrm{mg}$ EAG/100 g PF. Las especies silvestres mostraron los valores más altos, siendo estadísticamente diferente respecto a la variedad comercial. El contenido de polifenoles encontrado en este trabajo superó lo reportado por Wang y Lin. (2000), en cultivares comerciales de zarzamoras (204- 248 mg EAG/100 g PF) y frambuesas (208-267 mg EAG/100 g $\mathrm{PF}$ ) y concuerdan con lo reportado por Ramune et al. (2012) para 19 variedades mejoradas de frambuesas (278.6- $714.7 \mathrm{mg}$ EAG/100 g PF). Lo hallado en la especie silvestre Rubus hirsutus Thunb de 108. 23-269. $90 \mathrm{mg}$ EAG/100 g PF.

Respecto al contenido de flavonoides (Figura 1), osciló entre $93.13 \pm 1.7$ a $36.4 \pm 2.4 \mathrm{mg} \mathrm{EQ} / 100 \mathrm{~g}$ $\mathrm{PF}$, teniendo la menor cantidad las especies silvestres. Por otro lado, en el contenido de antocianinas destacaron las especies silvestres respecto a la variedad comercial, cuyas cantidades fluctuaron entre $18.43 \pm 1.05$ a $4.32 \pm 0.24 \mathrm{mg} \mathrm{L}^{-1}$, siendo $R$. adenotrichus y $R$. glaucus quienes reportaron la mayor cantidad de antocianinas, sin mostrar diferencia significativa entre ellas. Los valores de antocianinas encontrados en este estudio superan lo reportado para Rubus chamaemorus (Koponen et al., 2007) y al igual que lo mostrado en Rubus adentrichus (Martínez et al., 2011).

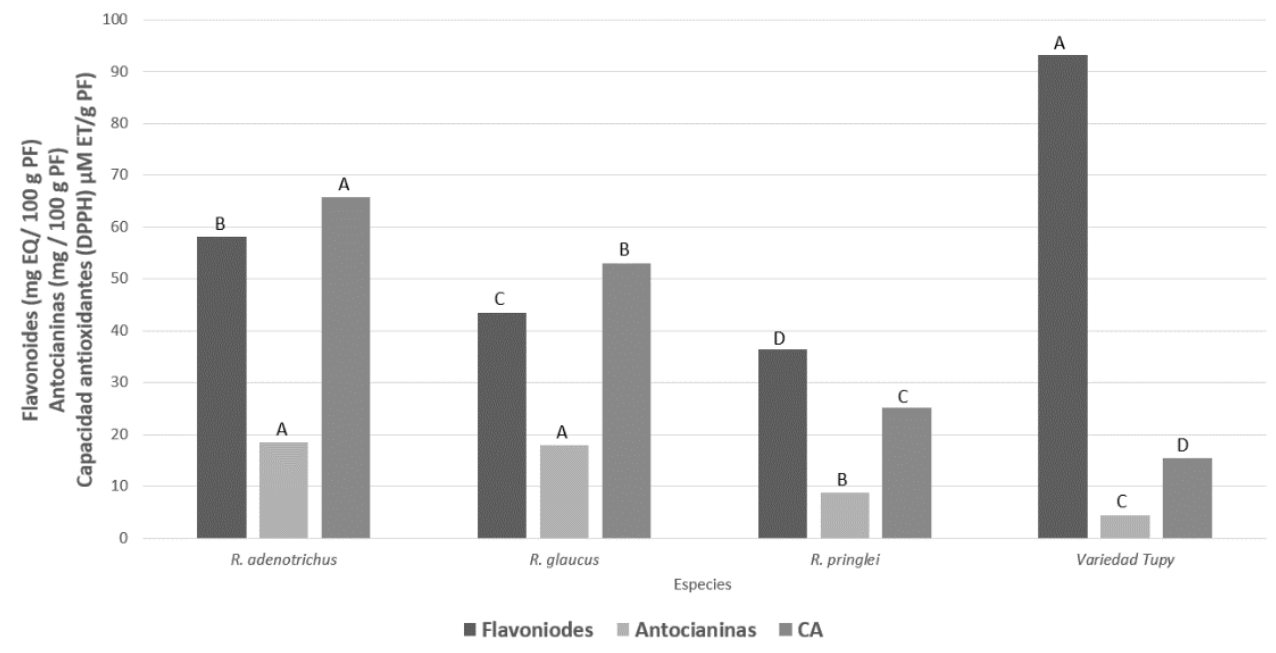

Figura 1. Concentración de flavonoides, antocianinas totales y capacidad antioxidante (CA) en frutos de tres especies silvestres del género Rubus y la variedad comercial Tupy ( \pm desviación estándar). Promedio con letras distintas indican diferencias significativas entre filas $(p \leq 0.05)$

Si bien han sido diversos los estudios que cuantifican los compuestos polifenólicos como los flavonoides y las antocianinas en las especies del género Rubus, se sabe que la síntesis de polifenoles está dada por factores como las condiciones de cultivo, especie y etapa fenólica de planta. Al igual su obtención puede verse condicionada por el proceso y el tipo de solvente utilizado (Valencia et al., 2013). Por lo que las especies analizadas en el presente trabajo pueden ser candidatas idóneas para la obtención de cultivares mejorados con alto contenidos de polifenoles. 
Por otro lado, se observó mayor potencial antioxidante en especies silvestres $(65.7 \pm 2.31-25.15$ $\pm 2.65 \mu \mathrm{M} \mathrm{ET} / \mathrm{g}$ PF) en comparación con la variedad Tupy (15.35 $\pm 1.01 \mu \mathrm{M} \mathrm{ET/g} \mathrm{PF),} \mathrm{esto}$ concuerda con lo encontrado en Rubus fructicosus y Rubus ideaous (25.3-35.5 $\mu \mathrm{M} \mathrm{ET/g} \mathrm{PF)} \mathrm{por}$ Paredes et al. (2010), al igual que lo mencionado por Cuevas et al. (2010), quienes reportaron que las especies silvestres del género Rubus poseen mayor contenido polifenólico y potencial antioxidante que la variedad comercial Tupy. Esto resultados evidencian la importancia de las especies silvestres mexicanas como fuentes de polifenoles y su posible incorporación a programas de producción antioxidantes con un objetivo industrial.

La capacidad antioxidante de los polifenoles se relaciona principalmente con características estructurales, tales como la presencia de dobles enlaces y grupos $O$-difenilo, hidroxilo o metoxi (Balasundram et al., 2006). La ausencia o sustitución de algunas de estas características estructurales reduce o inhibe la capacidad antioxidante. Skrovankova et al. (2015) mencionaron que la capacidad antioxidante de las zarzamoras está influenciada por la concentración del extracto. Sin embargo, Johnson et al. (2012), reportaron una relación estrecha entre la actividad antioxidante y el contenido de polifenoles.

Por otro lado, Hassimotto et al. (2008), evidenciaron que la eliminación de radicales está influenciada por el total de compuestos específicos tales como las antocianinas. En contraparte, Silva et al. (2007) demostraron que la relación entre la capacidad y la cantidad de polifenoles puede estar dada por la técnica implementada para la evaluación antioxidante del compuesto.

\section{Conclusiones}

En este estudio se demostró un alto contenido de sólidos solubles totales en las especies silvestres siendo candidatas para ser incorporadas en la elaboración de vinos y licores, además del alto contenido de polifenoles y capacidad antioxidante que presentaron sus extractos, cualidad valorada en la industria farmacéutica, además mostraron que cumplen con características fisicoquímicas apreciadas para su consumo en fresco. Lo anterior, subraya la importancia del rescate, utilización y estudios más detallados de las especies silvestres mexicanas del género Rubus, mismas que pueden ser material para la obtención de nuevas variedades comerciales, o bien para ser introducidas en programas de producción de metabolitos polifenólicos para la industria alimentaria o farmacéutica.

\section{Literatura citada}

Amid, M. and Manap, M. Y. A. 2014. Purification and characterisation of a novel amylase enzyme from red pitaya (Hylocereus polyrhizus) peel. Food Chem. 165(1):412-418.

AOAC. 2002. Association of Official Analytical Chemists. Official Methods of Analysis of Official Agricultural Chemist international. $21^{\text {th }}(\mathrm{Ed})$. San Antonio Tex. USA.

AOAC. 2007. Association of Official Analytical Chemists. Official Methods of Analysis of Official Agricultural Chemist International. 17 $7^{\text {th }}$ (Ed.). Current through revision \# 1. Gaithersburg, USA.

AOAC. 2007. Official methods of analysis of the Association of Official Agricultural Chemist. $18^{\mathrm{a}}$ (Ed). International. Gaithersburg, USA. 
Ayala, L. C.; Valenzuela, C. P. y Bohórquez, Y. 2013. Caracterización fisicoquímica de mora de castilla (Rubus glaucus benth) en seis estados de madurez. Biotecnología en el Sector Agropecuario y Agroindustrial. 11(2):10-18.

Azofeifa, G.; Boudard, F.; Morena, M.; Cristol, J.; Pérez, A. M.; Vaillant, F. and Michel, A. 2013. Antioxidant and anti-inflammatory in vitro activities of phenolic compounds from tropical highland blackberry (Rubus adenotrichus). J. Agric. Food Chem. 61(24):5798-5804.

Balasundram, N.; Sundram, K. and Samman, S. 2006. Phenolic compounds in plants and agriindustrial by-products: antioxidant activity, occurrence, and potential uses. Food Chemistry. 99(1)191-203.

Brand, W.; Cuvelier, M. E. and Berset, C. 1995. Use a free radical method to evaluate antioxidant activity. Lebensm Wiss Technology. 28(1):25-30.

Chang, C. C.; Yang, M. H.; Wen, H. M. and Chern, J. C. 2002 Estimation of total flavonoid content in propolis by two complementary colorimetric methods. J. Food Drug Analysis. 3(1):178-182.

Clark, J. R. and Finn, C. E. 2011. Blackberry breeding and genetics. Fruit, vegetable and cereal Sci. Biotechnol. 5(1):27-43.

Cuevas, R. E.; Dia, V. P.; Yousef, G. G.; García, S. P.; López, M. J.; Paredes, L. O.; González, de Mejía, E. and Lila, M. A. 2010. Inhibition of proinflamatory responses and antioxidant capacity of Mexican blackberry (Rubus spp.) extracts. J. Agric. Food Chem. 58(17):9542-9548.

Graham, J. and Woodhead, M. 2011. Rubus. In: Kole C. (eds) Wild crop relatives: genomic and breeding resources. Springer, Berlin, Heidelberg. DOI: doi.org/10.1007/978-3-64216057-8_9.

Hassimotto, N. M. A.; Mota, R. V. D.; Cordenunsi, B. R. and Lajolo, F. M. 2008. Physico-chemical characterization and bioactive compounds of blackberry fruits (Rubus sp.) grown in Brazil. Food Sci. Technol. (Campinas). 3(1):702-708.

Hummer, K. E. 2010. Rubus pharmacology: antiquity to the present. HortScience. 45(11):1587-1591.

Hummer, K. E. 2017. Blackberries: an introduction. Blackberries and their Hybrids. Crop Production Science in Horticulture. 26(1):152-167.

Johnson, M. H. and Mejia, E. G. 2012. Comparison of chemical composition and antioxidant capacity of commercially available blueberry and blackberry wines in Illinois. J. Food Sci. 77(1):141-148.

Koponen, J. M.; Happonen, A. M.; Mattila P. H. and Törrönen, R. 2007 Contents of anthocyanins and ellagitannins in selected foods consumed in Finland. J Agric Food Chem. 55(1):1612-1619.

Lewers, K. S.; Wang, S. Y. and Vinyard, B. T. 2010. Evaluation of blackberry cultivars and breeding selections for fruit quality traits and flowering and fruiting dates. Crop Sci. 50(6):2475-2491.

Martínez, N.; Arévalo, K.; Verde, M.; Rivas, C.; Oranday, A.; Núñez, M. y Morales, E. 2011. Antocianinas y actividad anti radicales libres de Rubus adenotrichus Schltdl (zarzamora). Rev. Mex. Cienc. Farmacéuticas. 42(1):4-7.

Paredes, L. O.; Cervantes, M. L. C.; Vigna, M. P. y Hernández T. P. 2010. Berries: improving human health and healthy aging and promoting quality life-a review. Plant Foods Human Nutr. 65(3):299-308. 
Petkovsek, M.; Schmitzer, V.; Slatnar, A.; Stampar, F. y Veberic, R. 2012. Composition of sugars, organic acids, and total phenolics in 25 wild or cultivated berry species. J. Food Sci. 77(10):1064-1070.

Quitmann, H.; Fan, R. and Czermak, P. 2014. Acidic organic compounds in beverage, food, and feed production. Adv. Biochem. Eng. Biotechnol. 143(1):91-141.

Segura, S.; Zavala, D.; Equihua, C.; Andrés, J. y Yepez, E. 2009. Los recursos genéticos de frutales en Michoacán. Rev. Chapingo Ser. Agric. 1583):297-305.

Silva, E. M.; Souza, J. N. S.; Rogez, H.; Rees, J. F. and Larondelle, Y. 2007. Antioxidant activities and polyphenolic contents of fifteen selected plant species from the Amazonian Region. Food Chem. 3(1):1012-1018.

Skrovankova, S.; Sumczynski, D.; Mlcek, J.; Jurikova, T. and Sochor, J. 2015. Bioactive compounds and antioxidant activity in different types of berries. Int. J. Mol. Sci. 16(10):24673-24706.

Tatjana, V.; Đurdina, R.; Radosav, C. and Gordana, S. M. 2010. Adventitious regeneration in blackberry (Rubus fruticosus L.) and assessment of genetic stability in regenerants. Plant Growth Regul. 61(3):265-275.

Valencia, S. E. y Guevara, P. A. 2013. Variación de la capacidad antioxidante y compuestos bioactivos durante el procesamiento del néctar de zarzamora (Rubus fructicosus L). Rev. Soc. Química del Perú. 79(2):116-125.

Vergara, M. F.; Vargas, J. y Acuña, J. F. 2016. Características físicoquímicas de frutos de mora de Castilla (Rubus glaucus Benth.) provenientes de cuatro zonas productoras de Cundinamarca, Colombia. Rev. Agron. Colomb. 3(1):336-345.

Wang, S. and Lin, H. S. 2000. Antioxidant activity in fruits and leaves of blackberry, raspberry and strawberry varieties with cultivar and developmental stage. J. Agric. Food Chem. 48(2):140-146.

Wrolstad, R. E.; Durst, R. W. and Lee, J. 2005. Tracking color in anthocyanins products. Trends in Food Sci. Technol. 16(9):423-428.

Zielinsli, H. and Kozolwska, H. 2000. Antioxidant activity and total phenolics in selected cereal grains and their different morphological fractions. J. Agric. Food Chem. 48(6):2008-2016. 